\title{
Momentum distribution of the uniform electron gas: improved parametrization and exact limits of the cumulant expansion
}

\author{
Paola Gori-Giorgi ${ }^{1,2}$ and Paul Ziesche ${ }^{2}$ \\ 1 INFM Center for Statistical Mechanics and Complexity, and Dipartimento di Fisica, \\ Università di Roma "La Sapienza", Piazzale A. Moro 2, I-00185 Rome, Italy \\ 2 Max-Planck-Institut für Physik komplexer Systeme, \\ Nöthnitzer Str. 38, D-01187 Dresden, Germany
}

(Dated: November 9, 2018)

\begin{abstract}
The momentum distribution of the unpolarized uniform electron gas in its Fermi-liquid regime, $n\left(k, r_{s}\right)$, with the momenta $k$ measured in units of the Fermi wave number $k_{\mathrm{F}}$ and with the density parameter $r_{s}$, is constructed with the help of the convex Kulik function $G(x)$. It is assumed that $n\left(0, r_{s}\right), n\left(1^{ \pm}, r_{s}\right)$, the on-top pair density $g\left(0, r_{s}\right)$ and the kinetic energy $t\left(r_{s}\right)$ are known (respectively, from accurate calculations for $r_{s}=1, \ldots, 5$, from the solution of the Overhauser model, and from Quantum Monte Carlo calculations via the virial theorem). Information from the high- and the low-density limit, corresponding to the random-phase approximation and to the Wigner crystal limit, is used. The result is an accurate parametrization of $n\left(k, r_{s}\right)$, which fulfills most of the known exact constraints. It is in agreement with the effective-potential calculations of Takada and Yasuhara [Phys. Rev. B 44, 7879 (1991)], is compatible with Quantum Monte Carlo data, and is valid in the density range $r_{s} \lesssim 12$. The corresponding cumulant expansions of the pair density and of the static structure factor are discussed, and some exact limits are derived.
\end{abstract}

PACS numbers: 71.10.Ca, 05.30.Fk

\section{INTRODUCTION}

In solid state theoryl and quantum chemistry, 2 the phenomenon of electron correlation and some of its details are hidden in the reduced densities and reduced

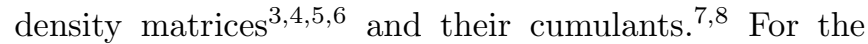
ground state of the uniform electron gas (jellium) of density $\rho=3 /\left(4 \pi r_{s}^{3}\right)$ (in a.u.), these quantities are the pair density $g\left(x, r_{s}\right)$ and the momentum distribution $n\left(k, r_{s}\right)$, where $k$ is measured in units of the Fermi wave number $k_{\mathrm{F}}=\left(3 \pi^{2} \rho\right)^{1 / 3}$ and $x$ is the scaled interelectronic distance, $x=k_{\mathrm{F}} r_{12}$.

Besides its relevance in the understanding of many effects in simple metals and semiconductors, the jellium model plays a crucial role in providing input quantities for approximate approaches to the many-electron problem of nonuniform density. Different approximate schemes, in fact, often need different quantities from jellium. As an example, density functional theory (DFT) uses the exchange-correlation energy of the uniform electron gas for the widely used local density approximation (LDA). For building nonempirical beyond-LDA function-

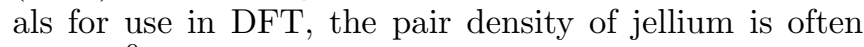
needed.

In such applications, the quantities from the uniform electron gas must be available in the form of analytic expressions. Since the jellium model is not exactly solvable, analytic expressions are built by interpolating between known exact limits, and by fitting the Quantum Monte Carlo (QMC) data, when available. Relevant examples are (i) the correlation energy used ip-thA implementation, built by using functional form $10,21,22$ which includes exact limits and interpolate the QMC data of Ceperley and Alder;13 (ii) the pair density, built by com- bining exact properties and fitting to QMC data, 14,15 or by simply interpolating between exact limits:16 (iii) the static local field factor, parametrized by fittinget QMC data of the static response: 18 (iv) the dynamical local field factors, built by using many known exact constraints.19 All these parametrized quantities are not strictly "exact", but are considered to be the closest to the true quantities, in the sense that this is the best one can presently obtain, and in the sense that they are accurate enough for the purpose for which they are needed.

In recent years, there has been increasing interest in a particular approach to the many-electron problem of nonuniform density, the so called density matrix functional theory (DMFT), which uses the one-body density matrix as basic variable. 0202122 Building a "local approximation" for DMFT is not an easy task: in a first attempt,23 the momentum distribution $n\left(k, r_{s}\right)$ of jellium has been used as input. Besides this important application, for which a reliable parametrization of $n\left(k, r_{s}\right)$ is needed, there are other reasons to focus on the momentum distribution of jellium. The definitions of "exchange" and "correlation" in DMFT are different from those in DFT and in Hartree-Fock-like approaches: in DMFT the cumulant partal of the pair density rises to be a key quantity. An accurate parametrization of $n\left(k, r_{s}\right)$ at metallic densities allows to extract the cumulant pair densitygf jellium, since the whole pair density is available $14,15,16$ The cumulant pair density can then be compared with recentattempts to calculate it in a high-density electron gas 24.25 and can be diagonalized in terms of "cumulant geminal "(faralog of "Overhauser geminals" for the pair density 26 27.28). Also, the study of exact limiting behaviors of the cumulant pair density is of great interest, since some of these limits could be 
approximately valid in nonuniform systems.

The momentum distribution of the uniform electron gas is also useful for the calculation of the exchangecorrelation correction to Compton profiles computed within LDA-DFT.29 Note also that $n\left(k, r_{s}\right)$ determines the part of the local-field factor beyond the randomphase approximation (RPA) which takes into account the change in occupation numbers in the Lindhard function [see Eq. (29) of Ref. 19].

The qualitative behavior of $n\left(k, r_{s}\right)$ is the following. It starts at $k=0$ with a value $n\left(0, r_{s}\right) \lesssim 1$ and decreases with increasing $k$. For $k<1$ it is concave. Then in the Fermi-liquid regime at $k=1$ there is a finite jump (Fermi gap) from $n\left(1^{-}, r_{s}\right)$ to a lower value $n\left(1^{+}, r_{s}\right)=$ $n\left(1^{-}, r_{s}\right)-z_{\mathrm{F}}\left(r_{s}\right)$ with logarithmic slopes at both sides of $k=1$. For $k>1$ (correlation tail) $n\left(k, r_{s}\right)$ is convex, and vanishes for $k \rightarrow \infty$. For $r_{s}=0$ (ideal Fermi gas) it is $n(k, 0)=\theta(1-k)$, where $\theta(x)$ is the Heaviside step function. Thus, the quasiparticle weight $z_{\mathrm{F}}\left(r_{s}\right)$ starts with $z_{\mathrm{F}}(0)=1$ and decreases with increasing interaction strength $r_{s}$. At large $r_{s}$ the electrons form a Wigner crystal with a smooth $n\left(k, r_{s}\right) . \quad r_{s} \ll 1$ and $r_{s} \gg 1$ are the weak- and strong-correlation limits, respectively. For intermediate values of $r_{s}$ a non-Fermi liquid regime may exist with $z_{\mathrm{F}}=0$. In such case $n\left(k, r_{s}\right)$ would be continuous vs. $k$, with a nonanalytical behavior at $k=1$.

In Ref. 30, the idea of using the convex Kulik function $G(x)$ to parametrize the two branches $(k<1$ and $k>1)$ of $n\left(k, r_{s}\right)$ is sketched. The function $G(x)$ appeared in Kulik' 31 RPA analysis of $n\left(k, r_{s}\right)$ near the Fermi edge $(|1-k|<<1)$, see Eq. (A4). $G(x)$ behaves as $c_{0}+c_{1} x \ln x$ for small $x$ (see Appendix A and Fig. (1), which corresponds to the correct nonanalytic behavior of $n\left(k, r_{s}\right)$ near the Fermi surface. So, supposing that the value at the centre, $n\left(0, r_{s}\right)$, and the values at the Fermi edge, $n\left(1^{-}, r_{s}\right)$ and $n\left(1^{+}, r_{s}\right)$, are known, it should be possible to represent $n\left(k, r_{s}\right)$ in terms of $G(x)$, with suitable prefactors and with suitable scaling (squeezing and stretching) of its argument. In this way, $n\left(k, r_{s}\right)$ becomes a functional of $n_{0}\left(r_{s}\right)=n\left(0, r_{s}\right)$, and of $n_{ \pm}\left(r_{s}\right)=n\left(1^{ \pm}, r_{s}\right)$, and can be designed to yield the proper normalization and the correct kinetic energy $t\left(r_{s}\right)$, which follows from the total energy $\epsilon\left(r_{s}\right)=t\left(r_{s}\right)+v\left(r_{s}\right)$ via the virial theorem. 32 In Ref 30 , the input data from Takada and Yasuhara (TY) 3334 for $n_{0}\left(r_{s}\right), n_{ \pm}\left(r_{s}\right)$ and $t\left(r_{s}\right)$ at $r_{s}=1, \ldots, 5$ have been used, together with the on-top pair density $g_{0}\left(r_{s}\right)=g\left(0, r_{s}\right)$ [which determines the large- $k$ behavior of $n(k)]$ from Ref. 27. The result is a field $n\left(k, r_{s}\right)$ for $r_{s} \in[1,6]$ which is correctly concave for $k<1$, convex for $k>1$, and with a Fermi gap $z_{\mathrm{F}}\left(r_{s}\right)$ at $k=1$ decreasing with growing $r_{s}$. The attempt to extend this procedure for $r_{s} \in[6,10]$ failed: $n\left(k<1, r_{s}\right)$ is no longer concave for $r_{s} \gtrsim 6$.

Here, an improved version of the parametrization of $n\left(k, r_{s}\right)$ in terms of the Kulik function $G(x)$ is presented. Our parametrized momentum distribution is in good agreement with the TY values, 3334 and is valid in the range of densities $r_{s} \lesssim 12$. Previous parametrizations of $n\left(k, r_{s}\right) 35.3637 .38$ used the Quantum Monte Carlo (QMC) data of Ref. 35 as an input. However, QMC data are presently only available for $0.4 \lesssim k \lesssim 0.9$ and $1.1 \lesssim k \lesssim$ 1.5 , thus not providing information about $n\left(k, r_{s}\right)$ near the centre, $k=0$, and at the Fermi edge, $k=1$. In these last regions, in fact, different parametrizations of the same QMC data can be rather different from each other. 55.36 .37 Our construction of $n\left(k, r_{s}\right)$ uses information from the effective-potential calculations of Takada and Yasuhara, 33 , 34 from the high- and low-density limits of $n\left(k, r_{s}\right)$, corresponding to RPA and the Wigner crystal (YC) limit, and from accurate parametrizations of $t\left(r_{s}\right) 12$ and of $g_{0}\left(r_{s}\right)$.27 In the regions where QMC data are available, our $n\left(k, r_{s}\right)$ is compatiple mith them. Also, with respect to previous works $35,36,37.38$ the functional form used here satisfies more exact limits. In particular, the logarithmic behavior at the Fermi edge is taken into account for the first time. 39 Notice that it causes the logarithmic divergence of $t\left(r_{s} \rightarrow 0\right)$. 40

Using our $n\left(k, r_{s}\right)$, the moments $\left\langle k^{\nu}\right\rangle$, the correlation entropy, and the one-body reduced density matrix $f\left(x, r_{s}\right)$ are evaluated. The latter appears in the cumulant partitioning of the pair density $g\left(x, r_{s}\right)$. The static structure factor $S\left(k, r_{s}\right)$, related to the pair density via Fourier transform, the particle-number fluctuations in fragments $\Delta N_{\Omega}\left(r_{s}\right)$, and the potential energy $v\left(r_{s}\right)$ are discussed in terms of their cumulant partitioning, and some exact limits are derived. Finally, by means, ff an accurate model for the spin-resolved pair density, 15 the cumulant part of $g\left(x, r_{s}\right)$ is extracted.

The paper is organized as follows. In Sec. II, the known sum rules and limiting cases for $n\left(k, r_{s}\right)$ are reported, and they are used in Sec. III to build up our parametrization of the momentum distribution via the Kulik function. Sec. IV is devoted to the calculation and discussion of different measures of the correlation strength, of the one-matrix, and of the cumulant expansion of the pairdensity. In Sec. $\mathrm{\nabla}$, we study the cumulant partitioning of the static structure factor, of the density fluctuations and of the potential energy. Conclusions and future developments are reported in the last Sec. VI.

\section{SUM RULES AND LIMITING CASES}

How is $n\left(k, r_{s}\right)$ defined? Starting from the many-body wave function $\Psi(1, \ldots, N)$, the one-body reduced density matrix (1-matrix for short) results from the $N-1$ contraction

$$
\begin{aligned}
& \gamma\left(1 \mid 1^{\prime}\right)=\int \frac{d 2 \ldots d N}{(N-1) !} \Psi(1,2, \ldots, N) \Psi^{*}\left(1^{\prime}, 2, \ldots N\right),(1) \\
& \int \frac{d 1 \ldots d N}{N !}|\Psi(1, \ldots, N)|^{2}=1,
\end{aligned}
$$

with the notation $1 \equiv\left(\underline{r}_{1}, \sigma_{1}\right)$. For the uniform electron gas, $\gamma\left(1 \mid 1^{\prime}\right)=\rho \delta_{\sigma_{1} \sigma_{1}^{\prime}} f\left(k_{\mathrm{F}}\left|\underline{r}_{1}-\underline{r}_{1}^{\prime}\right|, r_{s}\right)$ defines the dimensionless 1-matrix $f\left(x, r_{s}\right)$. Then its Fourier transform is 


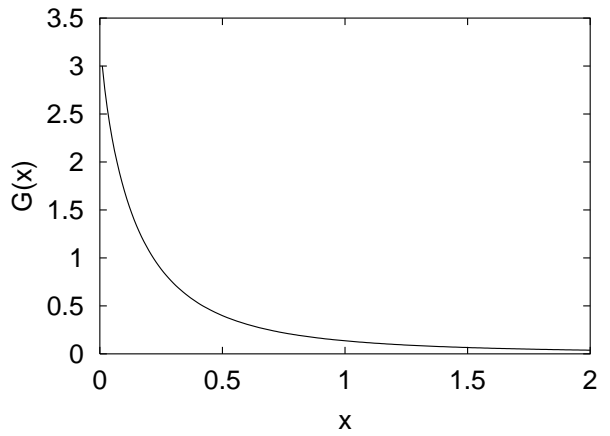

FIG. 1: The Kulik function $G(x)$ appearing in the RPA analysis of $n\left(k, r_{s}\right)$, see Eq. A4). At the origin $G(x)$ has a finite value, $G(0)=3.353337$ [Eq. (A7)], and a logarithmicdivergent slope, see Eq. A6.

the momentum distribution

$$
n\left(k, r_{s}\right)=\frac{\alpha^{3}}{2} \int_{0}^{\infty} d x^{3} \frac{\sin k x}{k x} f\left(x, r_{s}\right),
$$

where $d x^{3}=3 / 4 \pi d^{3} x=3 x^{2} d x$, and $\alpha=(4 / 9 \pi)^{1 / 3}$. $n\left(k, r_{s}\right)$ can be calculated using perturbation theory, directly with Green's functions 13 see Figs. 1a and $1 \mathrm{~b}$ of Ref. 24, or via the Hellmann-Feynman theorem 41 as the energy-derivative $n\left(k, r_{s}\right)=\delta E / \delta \varepsilon_{k}$, supposed $E$ is (perturbatively) knewn as a functional of $\varepsilon_{k}=\hbar k^{2} / 2 m$ and $v_{q}=4 \pi e^{2} / q^{2} .22$ Perturbative methods only work for high-densities, $r_{s} \ll 1$. At metallic and lower densities, other techniques must be used, namely, the effectivepotential method 33 which combines perturbation theory (Green's functions) with the Fermi-hymernetted chain approach, and the QMC simulations. 35.43 A more complete list of references concerning calculations and parametrizations can be found in Ref. 30 .

$n\left(k, r_{s}\right)$ has to satisfy the condition $0<n\left(k, r_{s}\right)<$ 1 (which guarantees the ensemble $N$-representability of the 1-matrix), and the sum rules ( $k$ in units of $k_{\mathrm{F}}$, and energies in ryd)

$$
\begin{aligned}
\int_{0}^{\infty} d k^{3} n\left(k, r_{s}\right) & =1, \\
\frac{1}{\left(\alpha r_{s}\right)^{2}} \int_{0}^{\infty} d k^{3} n\left(k, r_{s}\right) k^{2} & =t\left(r_{s}\right),
\end{aligned}
$$

where $t\left(r_{s}\right)$ can be written as the sum of the kinetic energy of the free Fermi gas, $3 / 5\left(\alpha r_{s}\right)^{-2}$, and of the kinetic energy of correlation, $t_{\mathrm{corr}}\left(r_{s}\right)$. For $r_{s} \ll 1, t_{\mathrm{corr}}\left(r_{s}\right)$ is known from RPA and from the lowest-order exchange diagram beyond it:44, 45,46 for a summary see Eq. (3.25) and Figs. 1a and 1b of Ref. 24. At larger $r_{s}, t_{\text {corr }}\left(r_{s}\right)$ can be obtained via the virial theorem 32 from parametrized QMC correlation energies.12

The large- $k$ behavior of $n\left(k, r_{s}\right)$ is determined by the kinks in the many-body wave-function which occur whenever two electrons are at contact or "on-top" (coalescing cusp properties), 44

$$
\begin{aligned}
n\left(k \rightarrow \infty, r_{s}\right) & =\frac{C\left(r_{s}\right)}{k^{8}}+O\left(\frac{1}{k^{10}}\right), \\
C\left(r_{s}\right) & =\frac{8}{9 \pi^{2}}\left(\alpha r_{s}\right)^{2} g_{0}\left(r_{s}\right),
\end{aligned}
$$

where $g_{0}\left(r_{s}\right)=g\left(0, r_{s}\right)$ is the on-top value of the pairdistribution function. In the $r_{s} \rightarrow$ Q limit, $g_{0}\left(r_{s}\right)$ can be obtained from perturbation theory, 4900 and at larger $r_{s}$ it has been calculated by solving an effective two-body Schrödinger equation.27.5

In a normal Fermi liquid, 52 the momentum distribution has a discontinuity and infinite slopes 53 at the Fermi edge, $k=1$,

$$
\begin{aligned}
n\left(k \rightarrow 1^{-}, r_{s}\right)= & n_{-}\left(r_{s}\right)-A\left(r_{s}\right)(1-k) \ln (1-k)+ \\
& O(1-k), \\
n\left(k \rightarrow 1^{+}, r_{s}\right)= & n_{+}\left(r_{s}\right)+A\left(r_{s}\right)(k-1) \ln (k-1)+ \\
& O(k-1) .
\end{aligned}
$$

In the following, $A\left(r_{s}\right)$ is referred to as the Fermi edge coefficient. In the small- $r_{s}$ limit, $n_{ \pm}\left(r_{s}\right)$ and $A\left(r_{s}\right)$ are known from RPA (see Appendix A). In the low-density or WC limit, the Fermi gap disappers $\left[n_{-}\left(r_{s}\right) \rightarrow n_{+}\left(r_{s}\right)\right]$, the infinite slopes at the Fermi edge may also vanish $\left[A\left(r_{s}\right) \rightarrow 0\right]$.

Near the cantre $k \rightarrow 0, n\left(k, r_{s}\right)$ should behave quadratically, 36.3738 .4

$$
n\left(k \rightarrow 0, r_{s}\right)=n_{0}\left(r_{s}\right)+B\left(r_{s}\right) k^{2}+O\left(k^{4}\right) .
$$

A simple argument in favour of Eq. (8) is that it holds both in the high- and in the low-density limit (see Appendix $A$ and $B$ ).

When $r_{s} \rightarrow 0$ exact results for $n\left(k, r_{s}\right)$ are known by means of RPA 142 (Appendix A). In the RPA treatment, the Kulik function $G(x)$ appears, 11 see Eqs. (A3)-(A5) and Fig. 1. $G(x)$ will be used in the next section to build up a parametrized $n\left(k, r_{s}\right)$ which satifies Eqs. (3)-(8).

In the low-density or strongly correlated limit, $r_{s} \rightarrow$ $\infty$, the electron gas undergoes Wigner crystallization (see, e.g., Refs. 54,56). A simple model for the momentum distribution in such regime is reported in Appendix B.

\section{IMPROVED PARAMETRIZATION OF $n\left(k, r_{s}\right)$}

The momentum distribution in terms of the Kulik function $G(x)$ of Fig. 11 is parametrized as follows. For $k<1$ we use the ansatz

$$
n_{<}\left(k, r_{s}\right)=n_{0}-\frac{\left[n_{0}-n_{-}\right]}{G(0)} G\left[x_{<}\left(k, r_{s}\right)\right],
$$

while for $k>1$ we use

$$
n_{>}\left(k, r_{s}\right)=\frac{n_{+}}{G(0)} G\left[x_{>}\left(k, r_{s}\right)\right],
$$


with $x_{<}\left(k, r_{s}\right)$ and $x_{>}\left(k, r_{s}\right)$ equal to

$$
\begin{aligned}
x_{<}\left(k, r_{s}\right)= & a \frac{\alpha r_{s}}{2 \pi^{2}} \frac{G(0)}{\left[n_{0}-n_{-}\right]} \frac{(1-k)}{\sqrt{4 \alpha r_{s} / \pi}}+ \\
& b \frac{\pi^{2}}{\alpha r_{s}} \sqrt{\frac{\pi}{3} \frac{(1-\ln 2)}{F^{\prime \prime}(0)} \frac{\left[n_{0}-n_{-}\right]}{G(0)} \frac{(1-k)^{2}}{k}(11)} \\
x_{>}\left(k, r_{s}\right)= & a \frac{\alpha r_{s}}{2 \pi^{2}} \frac{G(0)}{n_{+}} \frac{(k-1)}{\sqrt{4 \alpha r_{s} / \pi}}+ \\
& \sqrt{\frac{3 \pi(1-\ln 2)}{g_{0}} \frac{n_{+}}{G(0)}} \frac{\pi}{4 \alpha r_{s}}(k-1)^{4} .
\end{aligned}
$$

Here $F^{\prime \prime}(0)=17.968746$ [see Appendix A, Eq. (A1] ], and the $r_{s}$ dependence of $a, b, n_{0}, n_{ \pm}$and $g_{0}$ is not explictly shown for shortness. These constructions are such that $n_{<}(k) \rightarrow n_{0}, n_{-}$for $k \rightarrow 0,1^{-}$, respectively, and $n_{>}(k) \rightarrow n_{+}, 0$ for $k \rightarrow 1^{+}, \infty$, respectively. The behavior of the Kulik function for small and large arguments (see Appendix A) ensures the exact asymptotic expansion of Eqs. (5)-(8) near the centre, near the Fermi surface, and for large $k$.

The parameter $a\left(r_{s}\right)$ determines the Fermi edge coefficient $A\left(r_{s}\right)$ of the $|1-k| \ln |1-k|$ term at the Fermi surface,

$$
\begin{aligned}
n\left(k \rightarrow 1^{ \pm}, r_{s}\right)= & n_{ \pm}\left(r_{s}\right) \pm a\left(r_{s}\right)\left(\frac{\alpha r_{s}}{\pi}\right)^{1 / 2} \frac{1}{4}\left(\frac{\pi}{4}+\sqrt{3}\right) \times \\
& |1-k| \ln |1-k|+O(|1-k|) .
\end{aligned}
$$

The parameter $b\left(r_{s}\right)$ determines the curvature $B\left(r_{s}\right)$ of Eq. (8) at the centre, $k=0$,

$$
n\left(k \rightarrow 0, r_{s}\right)=n_{0}\left(r_{s}\right)-\frac{\pi^{4}}{\alpha^{2}} \frac{F^{\prime \prime}(0)}{2}\left[\frac{r_{s}}{b\left(r_{s}\right)}\right]^{2} k^{2}+O\left(k^{4}\right) .
$$

For small $r_{s}$ (RPA - Appendix A) it is $a\left(r_{s} \rightarrow 0\right)=1$ and $b\left(r_{s} \rightarrow 0\right)=1$.

In the preliminary version of Ref. 30, another (but similar) ansatz was introduced, and it was chosen $b\left(r_{s}\right)=1$. Two different functions, $a_{<}\left(r_{s}\right)$ and $a_{>}\left(r_{s}\right)$, for the coefficient of $|1-k| \ln |1-k|$ at $k=1^{-}$and $k=1^{+}$were fixed by the sum rules of Eqs. (3) and (41) (with $t_{\text {corr }}\left(r_{s}\right)$ from Ref. 12). The values $n_{0}\left(r_{s}\right)$ and $n_{ \pm}\left(r_{s}\right)$ were taken from the TY data (available for $r_{s}=1, \ldots, 5$ ). The on-top value $g_{0}\left(r_{s}\right)$ was taken from Ref. 27 .

In our improved ansatz of Eqs. (9)-(12), we set $a_{<}\left(r_{s}\right)=a_{>}\left(r_{s}\right)=a\left(r_{s}\right)$ (in agreement with Ref. 53; also Figs. 7 and 8 of Ref. 30 confirm this), and we use again $t_{\text {corr }}\left(r_{s}\right)$ from Ref. 12 and $g_{0}\left(r_{s}\right)$ from Ref. 27. Since we want to extend our results in the density range $6 \leq r_{s} \leq 10$, where there are no data available for $n_{0}\left(r_{s}\right)$ and $n_{ \pm}\left(r_{s}\right)$, we extract information from the extreme low-density limit (Wigner crystal - see Appendix B), by following an oversimplified version of the idea presented in Ref. 55.

We first build $n_{0}\left(r_{s}\right)$ by using a functional form which recovers the exact high-density limit, includes the Wigner

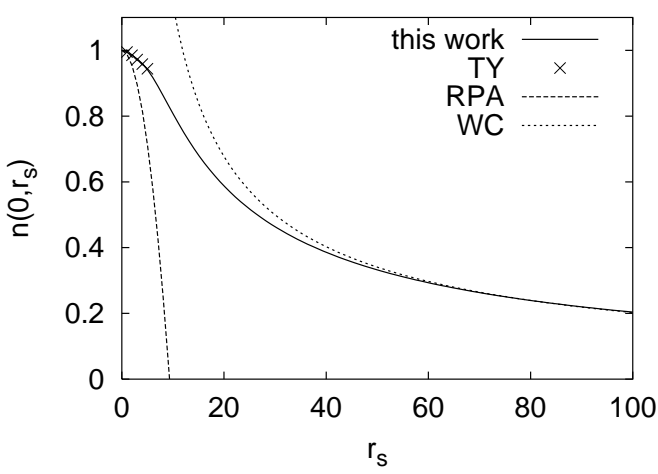

FIG. 2: Parametrized $n\left(0, r_{s}\right)$ (ge lid line), compared to the Takada-Yasuhara (TY) values 33,34 The high-density or RPA limit and the Wigner crystal (WC) limit are also shown.

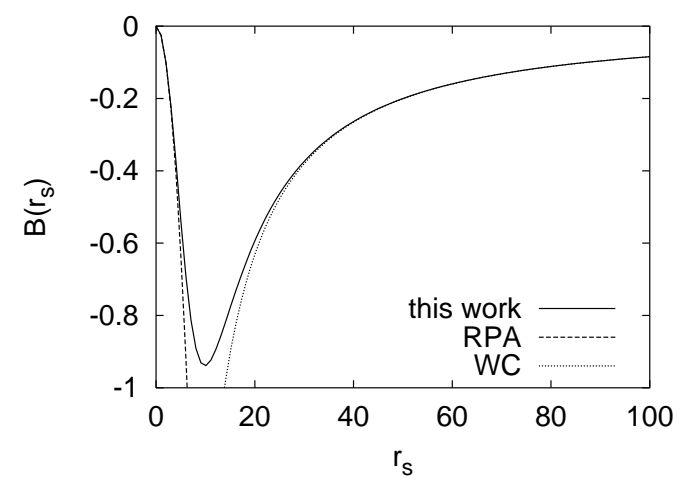

FIG. 3: Parametrized coefficient of the $k^{2}$ term near the centre $(k \rightarrow 0), B\left(r_{s}\right)=-\frac{\pi^{4}}{\alpha^{2}} \frac{F^{\prime \prime}(0)}{2}\left[\frac{r_{s}}{b\left(r_{s}\right)}\right]^{2}$. The high-density or $\mathrm{RPA}$ result, $b\left(r_{s} \rightarrow 0\right)=1$, and the Wigner crystal (WC) limit are also shown.

crystal behavior as $r_{s} \rightarrow \infty$, and has some free parameters to be fitted to the TY data. The result is reported in Fig. 2, together with the high- and low-density curves. It is given by

$$
n_{0}\left(r_{s}\right)=\frac{1+t_{1} r_{s}^{2}+t_{2} r_{s}^{5 / 2}}{1+t_{3} r_{s}^{2}+t_{4} r_{s}^{13 / 4}}
$$

with $t_{1}=0.003438169, t_{2}=0.00725313666, t_{3}=$ $0.014900367, t_{4}=0.00113244364\left(t_{1}-t_{3}\right.$ agrees with the RPA value $\left.-\left(\frac{\alpha}{\pi^{2}}\right)^{2} 4.1123=-0.01146\right)$. We then build the parameter $b\left(r_{s}\right)$ by a simple interpolation between the high- and low-density limit of the curvature at the centre (see Fig. 3). The result is

$$
b\left(r_{s}\right)=\left(1+0.0009376925 r_{s}^{13 / 4}\right)^{1 / 2} .
$$

Finally, the values at the Fermi edge, $n_{ \pm}\left(r_{s}\right)$, and the coefficient of the infinite slope at the Fermi edge, $a\left(r_{s}\right)$, are obtained by fitting the TY values for $n_{ \pm}\left(r_{s}\right)$ while imposing the normalization and the kinetic energy 12 sum rules of Eqs. (3) and (价. The results are parametrized 

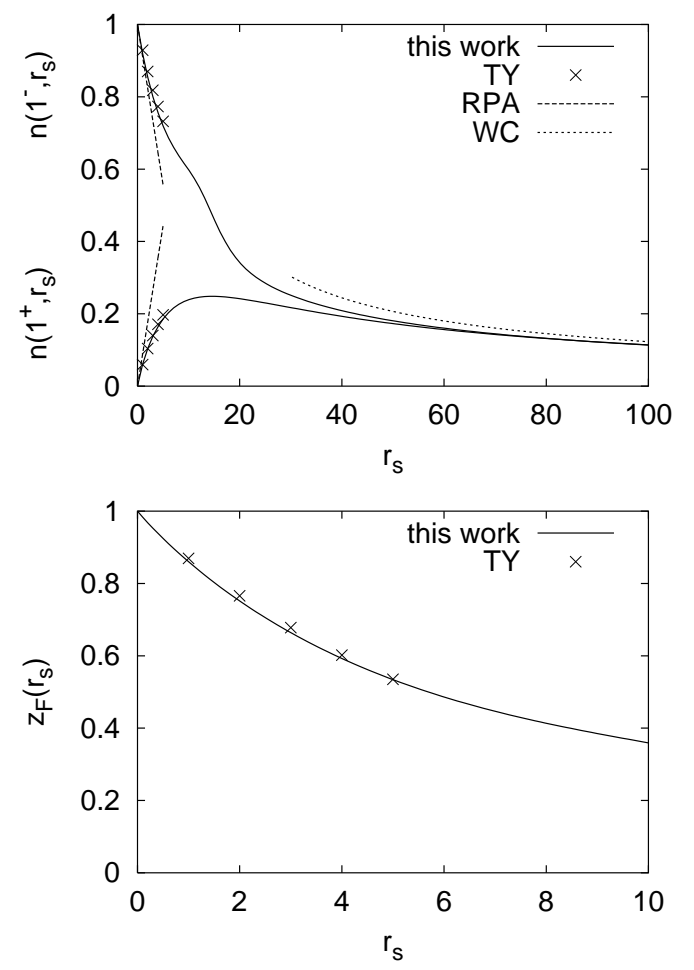

FIG. 4: Upper panel: parametrized $n\left(1^{ \pm}, r_{s}\right)$ (sqtiplines), compared to the Takada-Yasuhara (TY) values.33.34 The high-density or RPA limit and the Wigner crystal (WC) limit are also shown. Lower panel: value of the Fermi gap $z_{\mathrm{F}}\left(r_{s}\right)=n\left(1^{-}, r_{s}\right)-n\left(1^{+}, r_{s}\right)$ as a function of $r_{s}$; parametrization is compared with the TY results. 33.34

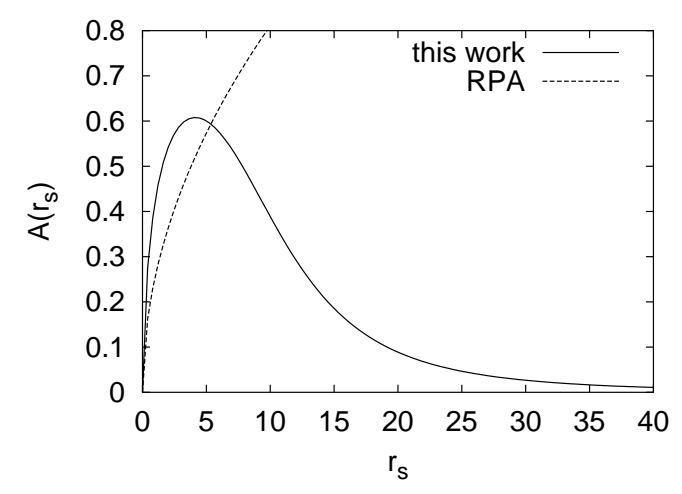

FIG. 5: Parametrized coefficient of the infinite slope at the Fermi edge, $A\left(r_{s}\right)=a\left(r_{s}\right)\left(\frac{\alpha r_{s}}{\pi}\right)^{1 / 2} 0.63$. The present result is compared with the RPA value, $a\left(r_{s} \rightarrow 0\right)=1$. Notice Ref. 39.

with the inclusion of the high- and low- density limits, and are equal to

$$
n_{-}\left(r_{s}\right)=\frac{1+v_{1} r_{s}+v_{2} r_{s}^{2}+v_{3} r_{s}^{3}}{1+v_{4} r_{s}+v_{5} r_{s}^{2}+v_{6} r_{s}^{3}+v_{7} r_{s}^{15 / 4}}
$$

with $v_{1}=-0.0679793, v_{2}=-0.00102846, v_{3}=$ $0.000189111, v_{4}=0.0205397, v_{5}=-0.0086838, v_{6}=$ $6.87109 \cdot 10^{-5}, v_{7}=4.868047 \cdot 10^{-5}\left(v_{1}-v_{4}\right.$ agrees with
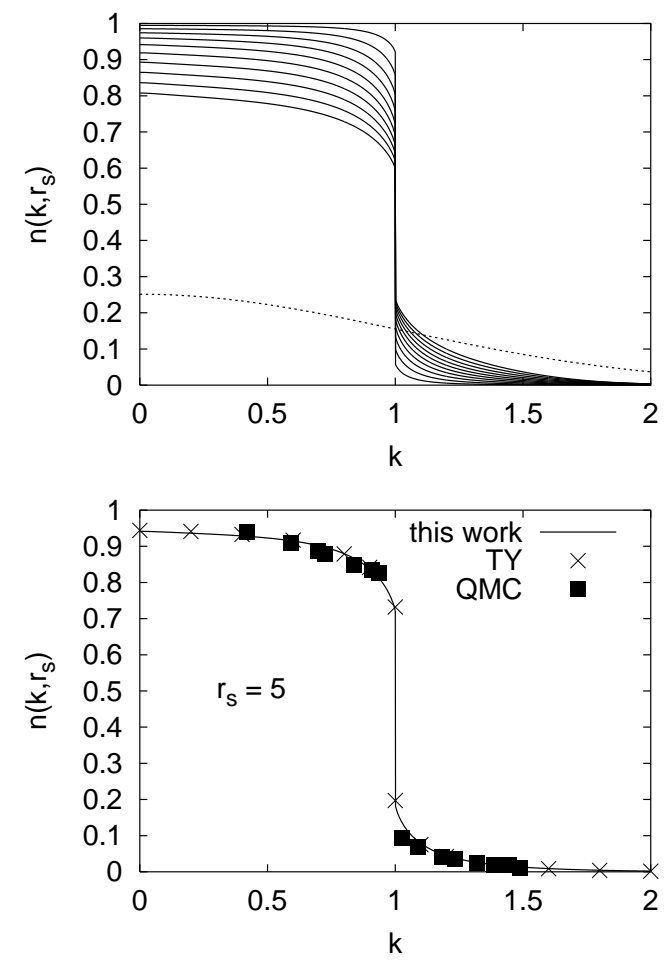

FIG. 6: Upper panel: momentum distribution calculated with Eqs. (9)-(12) for $r_{s}=1,2, \ldots, 10$ (solid lines). In the Wigner limit, $n\left(k, r_{s} \gg 1\right)$ is calculated with Eq. (B1) (dashed line, corresponding to $\left.r_{s}=75\right)$. Lower panel: cqmprison of the present work with the Takada-Yasuhara (TY) 33 momentum distribution and the QMC calculation of Ref. 35 for $r_{s}=5$.

the RPA value $\left.-\frac{\alpha}{2 \pi^{2}} 3.3533=-0.088519\right)$, and

$$
n_{+}\left(r_{s}\right)=\frac{q_{1} r_{s}}{1+q_{2} r_{s}^{1 / 2}+q_{3} r_{s}^{7 / 4}},
$$

with $q_{1}=0.088519$ (from RPA), $q_{2}=0.45, q_{3}=$ 0.022786335 ;

$$
a\left(r_{s}\right)=\frac{1+p_{1} r_{s}^{1 / 4}+p_{2} r_{s}^{1 / 2}}{1+p_{3} r_{s}^{1 / 4}+p_{4} r_{s}^{1 / 2}+p_{5} r_{s}+p_{6} r_{s}^{6}},
$$

with $p_{1}=-78.8682, p_{2}=-0.0989941, p_{3}=-68.5997$, $p_{4}=38.1159, p_{5}=-17.6829, p_{6}=-0.01136759$. Our parametrized $n\left(k, r_{s}\right)$ breaks down at $r_{s} \gtrsim 12$ [in the density range $12 \lesssim r_{s} \lesssim 16, n\left(k>1, r_{s}\right)$ is no longer convex, and for $r_{s} \gtrsim 16$ the unphysical result $n_{-}<n_{+}$ is obtained when the sum rules of Eqs. (3) and (蛋) are imposed].

In the upper panel of Fig. Q, we show the functions $n_{ \pm}\left(r_{s}\right)$, together with the TY values, and the high and low-density limits (here the $r_{s} \rightarrow \infty$ limit is considered to be the inflexion point of the $\mathrm{WC}$ momentum distribution, see Eq. (B4)). As said, our model is only valid for $r_{s} \lesssim$ 12 , so that $n_{-}\left(r_{s}\right)$ and $n_{+}\left(r_{s}\right)$ at densities lower than $r_{s}=12$ are no more obtained from the constraints of Eqs. (3) and (4). Thus, the strange behavior of $n_{-}\left(r_{s}\right)$ 


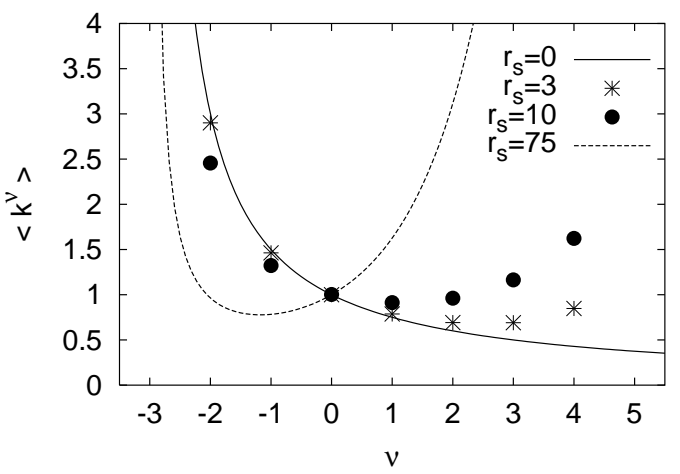

FIG. 7: The moments $\left\langle k^{\nu}\right\rangle$ of $n\left(k, r_{s}\right)$ for $r_{s}=3$ and $r_{s}=10$. The corresponding results for the noninteracting gas $\left(r_{s}=0\right)$ and for the Wigner crystal at $r_{s}=75$ are also reported.

at $r_{s} \sim 16$ does not affect our results. Also, the scheme presented here for the transition between the metallic and the extreme low-density region is oversimplified and must not be regarded as rigorous or reliable: we did not take into account the transition to the partially polarized electron gas (which affects the $r_{s} \gtrsim 50$ densities 56 ), as well as many other features. However, our results seem to be reliable in the relevant density range $r_{s} \lesssim 12$, and the simple picture of the upper panel of Fig. If is only a "naïve suggestion". In the lower panel of Fig. 目, we compare our parametrized $z_{\mathrm{F}}\left(r_{s}\right)$ with the TY results. In Fig. 5, we report the $r_{s}$ dependence of the Fermi edge coefficient $A\left(r_{s}\right)$. Finally, in Fig. 6, we present in the upper panel our parametrized $n\left(k, r_{s}\right)$ for $1 \leq r_{s} \leq 10$, and in the lower panel we compare our result with the TY $n\left(k, r_{s}\right)$ and with the QMC data of Ref. 35 for $r_{s}=5$.

\section{MOMENTS, CORRELATION ENTROPY, 1-MATRIX, AND CUMULANT EXPANSION}

With the now available momentum distribution $n\left(k, r_{s}\right)$, its moments

$$
\left\langle k^{\nu}\right\rangle=\int_{0}^{\infty} d k^{3} n\left(k, r_{s}\right) k^{\nu},
$$

can be evaluated in addition to the normalization for $\nu=$ 0 and the kinetic energy for $\nu=2$ [Eqs. (3) and (4)]. They are shown in Fig. 7 for $r_{s}=3$ and 10, together with the $r_{s}=0$ (ideal Fermi gas) and with the WC result $\left(r_{s}=75\right)$. The expression

$$
(\Delta t)^{2}=\frac{1}{\left(\alpha r_{s}\right)^{4}}\left[\left\langle k^{4}\right\rangle-\left\langle k^{2}\right\rangle^{2}\right]
$$

$\left(\right.$ measured in $\left.\mathrm{ryd}^{2}\right)$ describes the fluctuation of the kinetic energy. The moments $\left\langle k^{2}\right\rangle$ and $\left\langle k^{4}\right\rangle$ determine the small$x$ behavior of the 1-matrix, see Eq. (25).

In Refs. 57,58, the entropy-like expression $s\left(r_{s}\right)=$ $-\left\langle\ln n\left(k, r_{s}\right)\right\rangle$ as a function of the interaction strength $r_{s}$

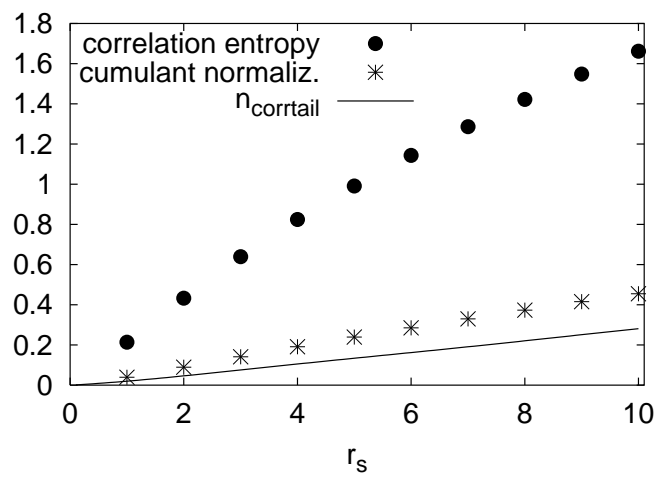

FIG. 8: The particle-hole symmetric correlation entropy [Eq. 22 ], the normalization of the cumulant pair density [r.h.s. of Eq. (34)], and the correlation tail normalization $n_{\text {corrtail }}\left(r_{s}\right)$ [Eq. (23)] as a function of $r_{s}$.

has been used as a measure of the correlation strength. 99 Here the expression

$$
\begin{aligned}
s_{\mathrm{ph}}\left(r_{s}\right)= & \int d k^{3}(-1)\left\{n\left(k, r_{s}\right) \ln n\left(k, r_{s}\right)+\right. \\
& {\left.\left[1-n\left(k, r_{s}\right)\right] \ln \left[1-n\left(k, r_{s}\right)\right]\right\} }
\end{aligned}
$$

is introduced as an alternative with the understanding that $n\left(k, r_{s}\right)$ and $1-n\left(k, r_{s}\right)$ are the probabilities for the momentum state $\underline{k}$ to be occupied (with spin up and spin down) and empty, respectively. The entropy of this probability 'distribution' is just the integrand of Eq. (22), and $s_{\mathrm{ph}}\left(r_{s}\right)$ is the sum of all these entropies. Notice its invariance under the exchange $n\left(k, r_{s}\right) \leftrightarrow 1-n\left(k, r_{s}\right)$, which is referred to as particle-hole symmetry in the ReducedDensity-Matrix community. This symmetry is an intrinsic property of the correlation energy as a functional of the 1-matrix $61 s_{\mathrm{ph}}\left(r_{s}\right)$ is plotted in Fig. 8. Another measure of the correlation strength is the correlation-tail normalization

$$
n_{\text {corrtail }}\left(r_{s}\right)=\int_{1}^{\infty} d k^{3} n\left(k, r_{s}\right),
$$

also reported in Fig. 8. For large $r_{s}$, the Fermi edge disappears, $z_{\mathrm{F}}\left(r_{s}\right)=0$, and also any relict of it, $A\left(r_{s}\right)=$ 0 , then the inflexion point of $n\left(k, r_{s}\right)$ vs. $k$ may serve in Eq. (23) as the lower limit.

With $n\left(k, r_{s}\right)$ also the (dimensionless) 1-matrix

$$
f\left(x, r_{s}\right)=\int_{0}^{\infty} d k^{3} \frac{\sin k x}{k x} n\left(k, r_{s}\right), \quad x=k_{\mathrm{F}}\left|\underline{r}-\underline{r}^{\prime}\right|
$$

is available as the inverse of Eq. (2). It has the small-x behavior

$$
\begin{aligned}
f\left(x \ll 1, r_{s}\right)= & 1-\frac{\left\langle k^{2}\right\rangle}{3 !} \cdot x^{2}+\frac{\left\langle k^{4}\right\rangle}{5 !} \cdot x^{4} \\
& -\frac{1}{5 !} \frac{2}{9 \pi}\left(\alpha r_{s}\right)^{2} g_{0}\left(r_{s}\right) \cdot x^{5}+O\left(x^{6}\right)(25)
\end{aligned}
$$




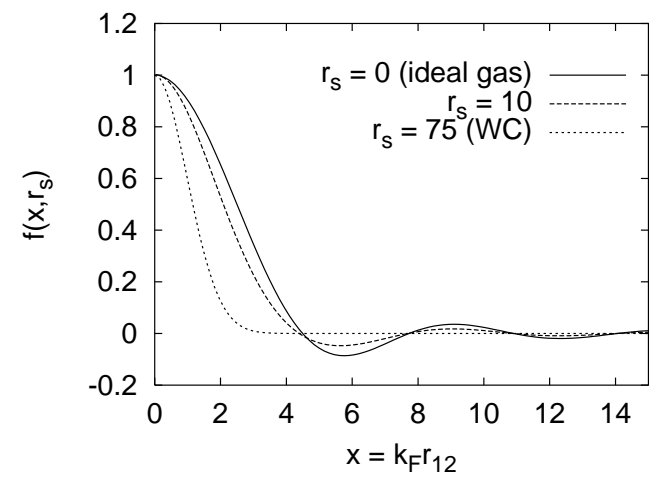

FIG. 9: 1-matrix $f\left(x, r_{s}\right)$ for $r_{s}=0$ (ideal Fermi gas), for $r_{s}=10$ (present model), and for $r_{s}=75$ [Wigner crystal (WC) limit, Eq. (B1)].

and the large- $x$ asymptotics (Friedel oscillations with reduced amplitudes)

$$
\begin{aligned}
f\left(x \gg 1, r_{s}\right)= & -3 z_{\mathrm{F}}\left(r_{s}\right) \frac{\cos x}{x^{2}}+\frac{3}{x^{3}}\left[z_{\mathrm{F}}\left(r_{s}\right) \sin x-\right. \\
& \left.\pi A\left(r_{s}\right) \cos x\right]+O\left(\frac{1}{x^{4}}\right),
\end{aligned}
$$

see Appendix G. $A\left(r_{s}\right)=a\left(r_{s}\right)\left(\frac{\alpha r_{s}}{\pi}\right)^{1 / 2} 0.63$ is the Fermi edge coefficient, the prefactor of the logarithmic term $(k-$ 1) $\ln |k-1|$ in $n\left(k \approx 1, r_{s}\right)$. The factor 0.63 is the Kulik number 7.91, see Eq. A6), divided by $4 \pi$. In the inverse Fourier transform (2), the oscillatory terms of Eq. (26) do not affect the small- $k$ behavior of $n\left(k, r_{s}\right)$, because their average is zero. Since $n\left(k \ll 1, r_{s}\right)=n_{0}\left(r_{s}\right)+$ $O\left(k^{2}\right)$, the large- $x$ behavior of the non-oscillatory $f\left(x, r_{s}\right)$ is $\propto 1 / x^{6}$ or faster. $f\left(x, r_{s}\right)$ is displayed in Fig. 9. One may partition $n\left(k, r_{s}\right)$ and correspondingly $f\left(x, r_{s}\right)$ in the following way:

$$
\begin{aligned}
& n\left(k, r_{s}\right)=z_{\mathrm{F}}\left(r_{s}\right) \theta(1-k)+n_{1}\left(k, r_{s}\right), \\
& f\left(x, r_{s}\right)=3 z_{\mathrm{F}}\left(r_{s}\right) \frac{j_{1}(x)}{x}+f_{1}\left(x, r_{s}\right),
\end{aligned}
$$

where $j_{1}(x)=(\sin x-x \cos x) / x^{2} \cdot n_{1}\left(k, r_{s}\right)$ is a continuous function with $n_{1}\left(1^{-}, r_{s}\right)=n_{1}\left(1^{+}, r_{s}\right)$ and an infinite slope at $k=1$. Figure 10 shows $n_{1}\left(k, r_{s}\right)$ and $f_{1}\left(x, r_{s}\right)$ for $r_{s}=5$.

The 1-matrix squared appears in the cumulant partitioning of the pair density,

$$
g\left(x, r_{s}\right)=1-\frac{1}{2}\left|f\left(x, r_{s}\right)\right|^{2}-h\left(x, r_{s}\right), \quad x=k_{\mathrm{F}} r_{12} .
$$

This defines the cumulant pair density $h\left(x, r_{s}\right)$, which is the diagonal of the cumulant 2-matrix $\chi\left(1\left|1^{\prime}, 2\right| 2^{\prime}\right)$. The spin-resolved version of Eq. 28 is

$$
\begin{aligned}
& g_{\uparrow \uparrow}\left(x, r_{s}\right)=1-\left|f\left(x, r_{s}\right)\right|^{2}-h_{\uparrow \uparrow}\left(x, r_{s}\right), \\
& g_{\uparrow \downarrow}\left(x, r_{s}\right)=1-h_{\uparrow \downarrow}\left(x, r_{s}\right)
\end{aligned}
$$

with $g\left(x, r_{s}\right)=\frac{1}{2}\left[g_{\uparrow \uparrow}\left(x, r_{s}\right)+g_{\uparrow \downarrow}\left(x, r_{s}\right)\right]$ and $h\left(x, r_{s}\right)=$ $\frac{1}{2}\left[h_{\uparrow \uparrow}\left(x, r_{s}\right)+h_{\uparrow \downarrow}\left(x, r_{s}\right)\right]$. Notice that the (generalized
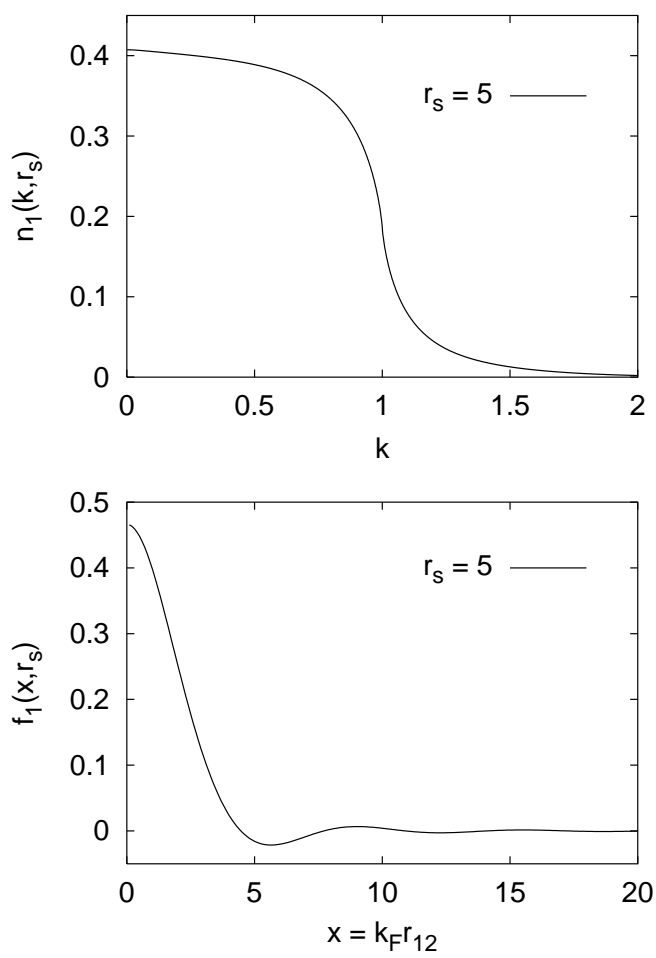

FIG. 10: Continuous part of $n\left(k, r_{s}\right), n_{1}\left(k, r_{s}\right)=n\left(k, r_{s}\right)-$ $z_{\mathrm{F}}\left(r_{s}\right) \theta(1-k)$ (upper panel), and the corresponding 1-matrix $f_{1}\left(x, r_{s}\right)$ (lower panel). The oscillations of $f_{1}(x)$ are due to the infinite slope of $n_{1}(k)$ at $k=1$.

exchange or) Fock term $\left|f\left(x, r_{s}\right)\right|^{2}$ appears only in the parallel-spin pair density and not in the antiparallel-spin pair density. $g_{\uparrow \uparrow}\left(x, r_{s}\right)$ describes the Fermi hole (due to both Pauli and Coulomb repulsion) with $g_{\uparrow \uparrow}\left(0, r_{s}\right)=$ $h_{\uparrow \uparrow}\left(0, r_{s}\right)=0$ and $g_{\uparrow \downarrow}\left(x, r_{s}\right)$ describes the Coulomb hole (only due to the Coulomb repulsion) with $g\left(0, r_{s}\right)<1$. In addition to the above mentioned correlation-strength indices, the quantities $h_{\uparrow \uparrow}^{\prime \prime}\left(0, r_{s}\right)$ measuring the on-top Fermi-hole curvature, and $h_{\uparrow \downarrow}\left(0, r_{s}\right)$ measuring the ontop Coulomb hole are other ones.

From Eq. (26) it follows

$$
\begin{aligned}
\left|f\left(x \gg 1, r_{s}\right)\right|^{2}= & \frac{9}{2}\left[\frac{z_{\mathrm{F}}^{2}\left(r_{s}\right)}{x^{4}}+\frac{2 \pi A\left(r_{s}\right) z_{\mathrm{F}}\left(r_{s}\right)}{x^{5}}\right](1+\cos 2 x) \\
& -9 \frac{z_{\mathrm{F}}^{2}\left(r_{s}\right)}{x^{5}} \sin 2 x+O\left(\frac{1}{x^{6}}\right)
\end{aligned}
$$

If this is inserted into Eq. (28), then the non-oscillatory terms, $\frac{9}{2} \frac{z_{\mathrm{F}}^{2}}{x^{4}}+\frac{9 \pi z_{\mathrm{F}} A}{x^{5}}$, are canceled by the asymptotics of $h\left(x, r_{s}\right)$, which follow from the sum-rule properties of the static structure factor $S\left(q, r_{s}\right)$, see Sec. V and Ref. 50 . The nominator of the oscillating $1 / x^{5}$ term can be written as

$$
\begin{aligned}
& 9 z_{\mathrm{F}} \sqrt{z_{\mathrm{F}}^{2}\left(r_{s}\right)+\pi^{2} A^{2}\left(r_{s}\right)} \cos \left(2 x+2 x_{0}\left(r_{s}\right)\right), \\
& \tan 2 x_{0}\left(r_{s}\right)=\frac{z_{\mathrm{F}}\left(r_{s}\right)}{\pi A\left(r_{s}\right)} .
\end{aligned}
$$



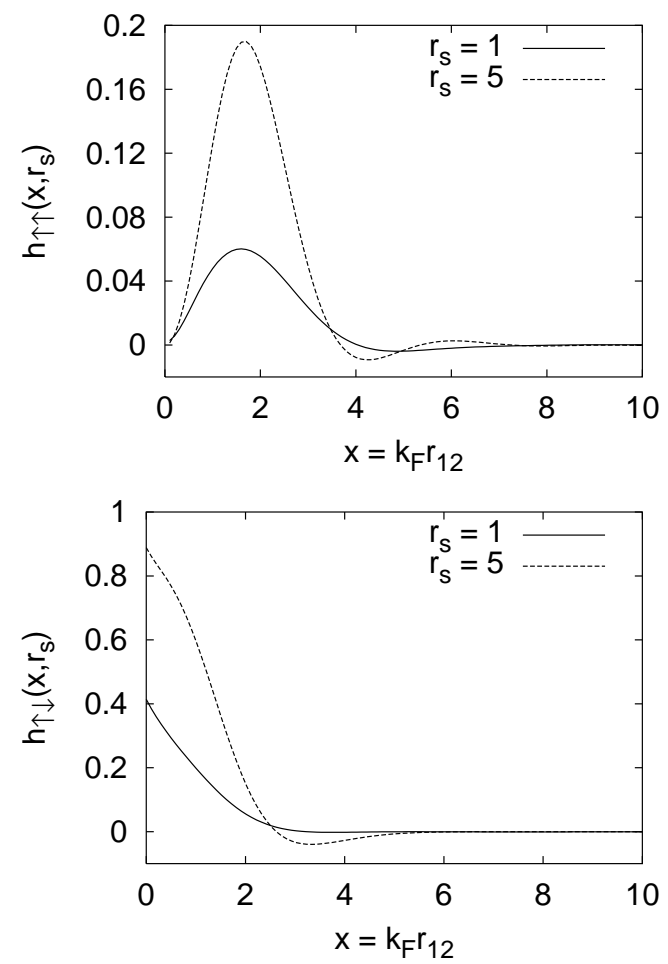

FIG. 11: Cumulant pair densities for parallel and antiparallel spins, obtained by combining the present work with the results of Ref. 15 .

The on-top properties of $g_{\uparrow \uparrow}\left(x \ll 1, r_{s}\right)$ and $g_{\uparrow \downarrow}\left(x \ll \frac{1}{1}, r\right)$ are determined by the coalescing cusp theorems.4762

With Eq. (29), with the spin-resolved pair densities of Ref. 15, and with $f\left(x, r_{s}\right)$ of this paper, the resulting cumulant pair densities $h_{\uparrow \uparrow}\left(x, r_{s}\right)$ and $h_{\uparrow \downarrow}\left(x, r_{s}\right)$ are plotted in Fig. 11. For small $r_{s}(\ll 1)$, our results agree with those of Ref. 24.

\section{STATIC STRUCTURE FACTOR, DENSITY FLUCTUATIONS, AND POTENTIAL ENERGY}

The cumulant partitioning of Eq. 28 causes corresponding decompositions of all the quantities containing $1-g\left(x, r_{s}\right)$. Such quantities are the static structure factor $S\left(q, r_{s}\right)$, the fluctuation $\Delta N_{\Omega}\left(r_{s}\right)$ of the particle number in a fragment $\Omega$, and the potential energy $v\left(r_{s}\right)$.

So, the static structure factor is given by

$$
S\left(q, r_{s}\right)=1-\frac{1}{2} \tilde{n}^{2}\left(q, r_{s}\right)-\tilde{h}\left(q, r_{s}\right)
$$

with the (generalized exchange or) Fock component

$$
\begin{aligned}
& \tilde{n}^{2}\left(q, r_{s}\right)=\alpha^{3} \int_{0}^{\infty} d x^{3} \frac{\sin q x}{q x}\left|f\left(x, r_{s}\right)\right|^{2}= \\
& \int_{0}^{\infty} d k^{3} n\left(k, r_{s}\right) \int_{-1}^{+1} d \zeta n\left(\sqrt{k^{2}+q^{2}-2 k q \zeta}, r_{s}(32)\right.
\end{aligned}
$$

(from which it follows that $\tilde{n}^{2}\left(q, r_{s}\right)$ has a discontinuity in its second derivative at $q=2$ ) and the cumulant component

$$
\tilde{h}\left(q, r_{s}\right)=\alpha^{3} \int_{0}^{\infty} d x^{3} \frac{\sin q x}{q x} h\left(x, r_{s}\right),
$$

which is simply the Fourier transform of the cumulant pair density $h\left(x, r_{s}\right)$. In Eq. (32) the convolution theorem has been applied. $\tilde{n}^{2}\left(q, r_{s}\right)$ is related to the probability of finding a pair of electrons with given relative momentum $q .27 .28$

Notice that the sum rule $S\left(q \rightarrow 0, r_{s}\right)=0$ is equivalent to the sum rule

$$
\alpha^{3} \int_{0}^{\infty} d x^{3} h\left(x, r_{s}\right)=\int_{0}^{\infty} d k^{3} n\left(k, r_{s}\right)\left[1-n\left(k, r_{s}\right)\right] .
$$

The l.h.s. equals $\tilde{h}\left(0, r_{s}\right)$ and the r.h.s. equals $1-$ $\frac{1}{2} \tilde{n}^{2}\left(0, r_{s}\right)$. It was already P.-Q Löwdin who has asked what meaning the r.h.s. has 63 According to Eq. (34), it fixes the normalization of the cumulant pair density $h\left(x, r_{s}\right)$ and is another particle-hole symmetric measure of the correlation strength; it is also reported in Fig. 8 . Eq. (34) is sometimes called perfect screening sum rule or charge neutrality condition.

For non-interacting electrons $\left(r_{s}=0\right)$, the cumulant part vanishes, $\tilde{h}\left(q, r_{s}\right)=0$, and the Fock part $S_{\mathrm{F}}\left(q, r_{s}\right)$ simply yields

$$
S_{0}(q)=\frac{q}{2}\left[\frac{3}{2}-\frac{1}{2}\left(\frac{q}{2}\right)^{2}\right] \theta\left(1-\frac{q}{2}\right)+\theta\left(\frac{q}{2}-1\right)
$$

with the linear small- $q$ behavior $3 q / 4$ For interacting electrons $\left(r_{s} \neq 0\right)$ the small- $q$ sum rule 5264

$$
S\left(q \ll 1, r_{s}\right)=\frac{1}{2\left(\alpha r_{s}\right)^{2} \omega_{\mathrm{pl}}\left(r_{s}\right)} \cdot q^{2}+O\left(q^{4}\right),
$$

and the large- $q$ sum rule 62

$$
S\left(q \gg 1, r_{s}\right)=1-\frac{8}{3 \pi} \alpha r_{s} g_{0}\left(r_{s}\right) \cdot \frac{1}{q^{4}}+O\left(\frac{1}{q^{6}}\right)
$$

hold. $\omega_{\mathrm{pl}}^{2}\left(r_{s}\right)=4 \pi e^{2} \rho / m=3 / r_{s}^{3}$ a.u. defines the plasma frequency.

The non-idempotency and the singularities of $n\left(k, r_{s}\right)$ determine the small- $q$ behavior of $S_{\mathrm{F}}\left(q, r_{s}\right)=1-$ $\frac{1}{2} \tilde{n}^{2}\left(q, r_{s}\right)$

$$
\begin{aligned}
S_{\mathrm{F}}\left(q \ll 1, r_{s}\right)= & S_{\mathrm{F}}\left(0, r_{s}\right)+\frac{3}{4} z_{\mathrm{F}}^{2}\left(r_{s}\right) q-A\left(r_{s}\right) z_{\mathrm{F}}\left(r_{s}\right) \times \\
& q^{2} \ln q+O\left(q^{2}\right),
\end{aligned}
$$

as shown in Appendix D. Notice that $S_{\mathrm{F}}\left(0, r_{s}\right)$ is equal to the r.h.s. of Eq. (34). In Fig. 12, we report $S_{\mathrm{F}}\left(q, r_{s}\right)$ for the ideal gas $\left(r_{s}=0\right)$, for $r_{s}=5$, and in the WC limit.

Eq. (38), together with the sum rule of Eq. (36), allows to extract the large- $x$ behavior of $h\left(x, r_{s}\right)$. Namely, 


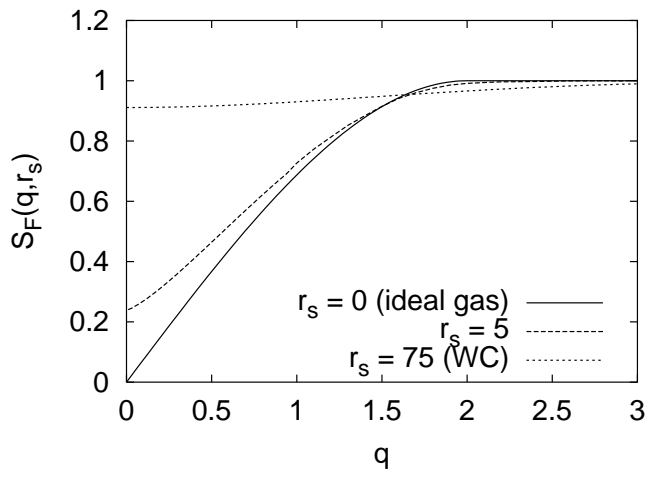

FIG. 12: The Fock component of the static structure factor for $r_{s}=0$ (ideal Fermi gas), $r_{s}=5$ (present work), and in the Wigner crystal (WC) limit for $r_{s}=75$.

because of Eq. (36), $\tilde{h}\left(q, r_{s}\right)$ must cancel both the linear and the $q^{2} \ln q$ term of $\tilde{n}^{2}\left(q, r_{s}\right)$. This implies that the large- $x$ behavior of the oscillation-averaged $\left\langle h\left(q, r_{s}\right)\right\rangle$ is:

$\left\langle h\left(x \gg 1, r_{s}\right)\right\rangle=-\frac{9}{4} \frac{z_{\mathrm{F}}^{2}\left(r_{s}\right)}{x^{4}}-\frac{9}{2} \frac{\pi A\left(r_{s}\right) z_{\mathrm{F}}\left(r_{s}\right)}{x^{5}}+O\left(\frac{1}{x^{6}}\right)$.

In Eq. (28) these terms cancel with the non-oscillatory long-range part of $\frac{1}{2}\left|f\left(x, r_{s}\right)\right|^{2}$.

If we consider within the uniform electron gas a certain fragment $\Omega$ (e.g., a sphere of radius $R$ ) containing on average $N_{\Omega}=\Omega /\left(4 \pi r_{s}^{3} / 3\right)=\left(R / r_{s}\right)^{3}$ electrons, and ask for the particle-number fluctuation $\Delta N_{\Omega}$ then the answer is 65.66

$$
\begin{gathered}
\frac{\left(\Delta N_{\Omega}\right)^{2}}{N_{\Omega}}=1- \\
\frac{\left(\alpha r_{s}\right)^{6}}{N_{\Omega}} \int_{\Omega} d^{3} x_{1} \int_{\Omega} d^{3} x_{2}\left[\frac{1}{2}\left|f\left(x, r_{s}\right)\right|^{2}+h\left(x, r_{s}\right)\right]=1- \\
\frac{\left(\alpha r_{s}\right)^{6}}{N_{\Omega}} \frac{3}{8 \pi} \int d^{3} q\left|\int_{\Omega} d^{3} x \mathrm{e}^{\mathrm{i} \underline{q} \underline{x}}\right|^{2}\left[\frac{1}{2} \tilde{n}^{2}\left(q, r_{s}\right)+\tilde{h}\left(q, r_{s}\right)\right],(40) \\
x=\left|\underline{x}_{1}-\underline{x}_{2}\right| .
\end{gathered}
$$

Again one may ask how differently the Fock and the cumulant parts contribute to their sum and to the conclusion 'correlation suppresses fluctuations' 1,66 In the case of a sphere $\Omega=4 \pi R^{3} / 3$, the term in the modulus in Eq. (40) is just $\Omega 3 j_{1}(q R) /(q R)$, so that the Fock term yields

$$
\begin{aligned}
& {\left[\frac{\left(\Delta N_{\Omega}\right)^{2}}{N_{\Omega}}\right]_{\mathrm{F}}=} \\
& 1-\frac{3}{2} \pi^{2} \Omega\left(\alpha r_{s}\right)^{9} \int_{0}^{\infty} d q^{3}\left[\frac{3 j_{1}(q R)}{q R}\right]^{2} \tilde{n}^{2}\left(q, r_{s}\right)(41)
\end{aligned}
$$

Also, the potential energy $v\left(r_{s}\right)$ consists of a Fock and a cumulant part: :

$$
\begin{aligned}
v\left(r_{s}\right) & =-\frac{\alpha^{2}}{r_{s}} \int_{0}^{\infty} d x^{3}\left[\frac{1}{2}\left|f\left(x, r_{s}\right)\right|^{2}+h\left(x, r_{s}\right)\right] \frac{1}{x} \\
& \left.=-\frac{\alpha^{2}}{r_{s}} \frac{3}{2} \int_{0}^{\infty} d q^{3}\left[\frac{1}{2} \tilde{n}^{2}\left(q, r_{s}\right)+\tilde{h}\left(q, r_{s}\right)\right] \frac{1}{q^{2}} 42\right)
\end{aligned}
$$

(in ryd). The Fock part can also be written ast

$$
\begin{aligned}
v_{\mathrm{F}}\left(r_{s}\right)= & -\frac{3}{2 \pi \alpha r_{s}} \int_{0}^{\infty} d k n\left(k, r_{s}\right) \int_{0}^{\infty} d k^{\prime} n\left(k^{\prime}, r_{s}\right) \times \\
& k k^{\prime} \ln \frac{k+k^{\prime}}{\left|k-k^{\prime}\right|}
\end{aligned}
$$

In lowest order, with $n\left(k, r_{s}\right) \rightarrow \theta(1-k)$, Eq. (43) yields $-3 /\left(2 \pi \alpha r_{s}\right)$. The logarithmic term of $v\left(r_{s} \rightarrow 0\right)$ arises from $v_{\mathrm{C}}\left(r_{s}\right)$, not from $v_{\mathrm{F}}\left(r_{s}\right)$.24

\section{SUMMARY AND OUTLOOK}

In Ref. 30, it was shown that the convex Kulik function $G(x)$, decorated with appropriate prefactors and with an appropriate inhomogeneous scaling of its argument, reproduces the momentum distribution $n\left(k, r_{s}\right)$ of the unpolarized uniform electron gas of density $\rho=3 / 4 \pi r_{s}^{3}$ in the metallic-density regime $r_{s} \in[1,6]$. The $r_{s}$-functions $n\left(0, r_{s}\right), n\left(1^{ \pm}, r_{s}\right)$, the on-top pair density $g\left(0, r_{s}\right)$, and the kinetic energy $t\left(r_{s}\right)$ form the input for such construction. In this work, we improved the parametrization of $n\left(k, r_{s}\right)$ via the Kulik function, and we extended it up to $r_{s} \lesssim 12$, including the high-density regime [Eqs. (9)-(12), and Fig. 6].

The Fourier transform of $n\left(k, r_{s}\right)$ yields the one-body reduced density matrix $f\left(x, r_{s}\right)$ (Figs. 9 and 10), with large $x$-oscillations arising from the Fermi gap $z_{\mathrm{F}}\left(r_{s}\right)$ and the Fermi edge coefficient $A\left(r_{s}\right)$, the prefactor of the logarithmic term in $n\left(k \approx 1, r_{s}\right)$, which is included in our parametrization for the first time (Fig. 5). Several measures of the correlation strength have been discussed (Fig. 8). With reliable models for the pair density, $g\left(x, r_{s}\right)$, the cumulant pair density $h\left(x, r_{s}\right)=1-$ $\frac{1}{2}\left|f\left(x, r_{s}\right)\right|^{2}-g\left(x, r_{s}\right)$ has been extracted (Fig. 11), as a prestep of its diagonalization in terms of cumulant geminals (analog with the diagonalization of the pair density in terms of Overhauser geminals). Future work also includes the generalization to the partially polarized gas. In this case, with $\zeta=\left(N_{\uparrow}-N_{\downarrow}\right) / N$, one has to consider different cases. For spin polarization $\zeta$ between 0 and 1 , two momentum distributions are to be described, $n_{\uparrow}\left(k, r_{s}, \zeta\right)$ for the spin-up electrons and $n_{\downarrow}\left(k, r_{s}, \zeta\right)$ for the spin-down electrons. So far, only the input data $g_{0}\left(r_{s}, \zeta\right)^{27}$ and $t\left(r_{s}, \zeta\right)^{12}$ are available in this more general case.

A small FORTRAN subroutine, which numerically evaluates our parametrized $n\left(k, r_{s}\right)$, is available at http://axtnt2.phys.uniroma1.it/PGG/elegas.html. 


\section{Acknowledgments}

The authors thank $\mathrm{Y}$. Takada for providing the data for $n\left(0, r_{s}\right)$ and $n\left(1^{ \pm}, r_{s}\right)$, and J. Cioslowski and G. Diener for helpful discussions. One author (P.G.-G.) gratefully aknowledges hospitality at the Max Planck Institut für Physik komplexer Systeme of Dresden (Germany), the other author (P.Z.) thanks P. Fulde for supporting this work.

\section{APPENDIX A: RANDOM PHASE APPROXIMATION}

In RPA it is 31, $42 n\left(k, r_{s}\right)=1-\left(\alpha r_{s} / \pi^{2}\right)^{2} H(k, 1)$ for $k<1$ and $\left(\alpha r_{s} / \pi^{2}\right)^{2} H(k, 1)$ for $k>1$, where

$$
\begin{aligned}
H(x<1, y)= & \frac{1}{x}\left\{\int_{1-x}^{1+x} \frac{d q}{q} \int_{0}^{\infty} d u\left[\frac{\frac{1-x^{2}}{2 q}}{\left(\frac{1-x^{2}}{2 q}\right)^{2}+u^{2}}-\frac{\frac{q}{2}+x}{\left(\frac{q}{2}+x\right)^{2}+u^{2}}\right] \frac{Q(q, u)}{q^{2}+y \frac{\alpha r_{s}}{\pi^{2}} Q(q, u)}\right. \\
& \left.+\int_{1+x}^{\infty} \frac{d q}{q} \int_{0}^{\infty} d u\left[\frac{\frac{q}{2}-x}{\left(\frac{q}{2}-x\right)^{2}+u^{2}}-\frac{\frac{q}{2}+x}{\left(\frac{q}{2}+x\right)^{2}+u^{2}}\right] \frac{Q(q, u)}{q^{2}+y \frac{\alpha r_{s}}{\pi^{2}} Q(q, u)}\right\} \\
H(x>1, y)= & \frac{1}{x} \int_{x-1}^{x+1} \frac{d q}{q} \int_{0}^{\infty} d u\left[\frac{\frac{x^{2}-1}{2 q}}{\left(\frac{x^{2}-1}{2 q}\right)^{2}+u^{2}}-\frac{x-\frac{q}{2}}{\left(x-\frac{q}{2}\right)^{2}+u^{2}}\right] \frac{Q(q, u)}{q^{2}+y \frac{\alpha r_{s}}{\pi^{2}} Q(q, u)}
\end{aligned}
$$

and

$$
Q(q, u)=2 \pi\left\{1+\frac{1+u^{2}-q^{2} / 4}{2 q} \ln \frac{(1+q / 2)^{2}+u^{2}}{(1-q / 2)^{2}+u^{2}}-u\left[\arctan \frac{1+q / 2}{u}+\arctan \frac{1-q / 2}{u}\right]\right\} .
$$

For small or large $k$ (far from the Fermi edge) it is $H(k, 1) \rightarrow F(k)$ with $F(k)=H(k, 0) . \quad F(k)$ has the small and large $k$ properties

$$
F(k \ll 1)=4.112335+8.984373 \cdot k^{2}+O\left(k^{4}\right)
$$

and

$$
F(k \gg 1)=\frac{8 \pi^{2}}{9} \cdot \frac{1}{k^{8}}+O\left(\frac{1}{k^{10}}\right),
$$

respectively 2021 The coefficient of $1 / k^{8}$ is 8.77298 . For $k$ near the Fermi edge it is 2021

$$
H(k, 1) \rightarrow \frac{\pi^{2}}{2 \alpha r_{s}} \frac{1}{k^{2}} G\left(\frac{|k-1|}{\sqrt{4 \alpha r_{s} / \pi}}\right)
$$

with

$$
G(x)=\left.\int_{0}^{\infty} d u \frac{R^{\prime}(u)}{R(u)} \cdot \frac{u}{u+y} \cdot \frac{R(u)-R(y)}{u-y}\right|_{y=x / \sqrt{R(u)}}
$$

and

$$
R(u)=1-u \arctan \frac{1}{u} .
$$

$G(x)$ has the small- $x$ behavior 31

$G(x \ll 1)=G(0)+\left[\pi\left(\frac{\pi}{4}+\sqrt{3}\right) \cdot x+O\left(x^{2}\right)\right] \ln x+O(x)$ with

$$
G(0)=\int_{0}^{\infty} d u(-1) \frac{R^{\prime}(u)}{R(u)} \cdot \arctan \frac{1}{u} \approx 3.353337 .
$$

The coefficient of $x \ln x$ is 7.908799 (the Kulik number). $G(x)$ has the large- $x$ behavior

$$
G(x \gg 1)=\frac{\pi}{6}(1-\ln 2) \cdot \frac{1}{x^{2}}+O\left(\frac{1}{x^{4}}\right) .
$$

The coefficient of $1 / x^{2}$ is 0.160668 (the Macke number). The Kulik function $G(x)$ is shown in Fig. 1

\section{APPENDIX B: THE MOMENTUM DISTRIBUTION OF THE WIGNER CRYSTAL}

In the low-density (large $r_{s}$ ) or strongly correlated limit, the electrons localize 5 and form a ferromagnetic body-centered cubic lattice with ap electrostatic (or Madelung) energy of $-1.792 / r_{s}$ ryd 67 The next term, $+2.65 / r_{s}^{3 / 2}$ ryd, describes the coupled harmonic zerotemperature motion in lowest order .66.69.70 To estimate the corresponding $n\left(k, r_{s}\right)$, we define with $3 \hbar \omega / 2=$ $2.65 / r_{s}^{3 / 2}$ (in ryd, or $\omega=0.88 / r_{s}^{3 / 2}$ in a.u.) the frequency of indepent oscillating electrons (Einstein model). So, from the momentum distribution of the harmonicoscillator ground state it follows

$$
n\left(k, r_{s} \rightarrow \infty\right)=\frac{4 \pi}{3} \frac{1}{\left(\pi \omega / k_{\mathrm{F}}^{2}\right)^{3 / 2}} \mathrm{e}^{-\frac{k^{2}}{\omega / k_{\mathrm{F}}^{2}}}
$$


see Refs. 71,72, and 73, p. 19. Note that $k$ is dimensionless (measured in units of $k_{\mathrm{F}}$ ) and that $\omega / k_{\mathrm{F}}^{2}=$ $0.88 \cdot \alpha^{2} r_{s}^{1 / 2}=0.24 \cdot r_{s}^{1 / 2}$. In Ref. 72, the factor 1 is used instead of 0.88 . $n\left(k, r_{s} \rightarrow \infty\right)$ is correctly normalized and yields with Eq. (4) the kinetic energy (in ryd)

$$
t\left(r_{s} \rightarrow \infty\right)=\frac{1}{2} \cdot \frac{2.65}{r_{s}^{3 / 2}}+\cdots
$$

as it should. The corresponding potential energy is

$$
v\left(r_{s} \rightarrow \infty\right)=-\frac{1.792}{r_{s}}+\frac{1}{2} \cdot \frac{2.65}{r_{s}^{3 / 2}}+\cdots .
$$

The inflexion-point trajectory with $r_{s}$ as parameter is described by (see left panel of Fig. 1)

$$
\begin{aligned}
& k_{\mathrm{infl}}\left(r_{s}\right)=\left(\omega /\left(2 k_{\mathrm{F}}^{2}\right)\right)^{1 / 2}=0.35 \cdot r_{s}^{1 / 4}, \\
& n_{\mathrm{infl}}\left(r_{s}\right)=\frac{4 \pi}{3} \frac{e^{-1 / 2}}{\left(\pi \omega / k_{\mathrm{F}}^{2}\right)^{3 / 2}}=\frac{3.88}{r_{s}^{3 / 4}}
\end{aligned}
$$

The region $k>k_{\text {infl }}$ (maybe to be referred to as correlation tail) contributes to the normalization the constant amount

$$
\int_{k_{\mathrm{infl}}}^{\infty} d k^{3} n\left(k, r_{s}\right)=\operatorname{Erf}\left(\frac{1}{\sqrt{2}}\right)-\sqrt{\frac{2}{\mathrm{e} \pi}}=0.80 .
$$

From Eq. (B1) it follows for $n\left(0, r_{s} \rightarrow \infty\right)$

$$
n_{0}\left(r_{s} \rightarrow \infty\right)=\frac{4}{3 \pi^{1 / 2}}\left(\frac{1}{0.24 r_{s}^{1 / 2}}\right)^{3 / 2}=\frac{6.40}{r_{s}^{3 / 4}}
$$

see Fig. 2; and for the curvature at the centre (the coefficient of $k^{2}$ )

$$
-\frac{4}{3 \pi^{1 / 2}} \frac{1}{\left(0.88 \alpha^{2} r_{s}^{1 / 2}\right)^{5 / 2}}=-\frac{26.71}{r_{s}^{5 / 4}}
$$

see Fig. 3 .

A more refined treatment takes into account that in harmonic approximation there are two transversal branches of harmonic lattice vibrations, $\omega_{t_{1,2}}\left(\underline{q}, r_{s}\right)$, and one longitudinal branch $\omega_{l}\left(\underline{q}, r_{s}\right)$ in the face-centred cubic Brillouin zone, satisfying the sum rule $\omega_{t_{1}}^{2}\left(\underline{q}, r_{s}\right)+$ $\omega_{t_{2}}^{2}\left(\underline{q}, r_{s}\right)+\omega_{l}^{2}\left(\underline{q}, r_{s}\right)=\omega_{\mathrm{pl}}^{2}\left(r_{s}\right)$. For $q=\overline{0}$ it is $\omega_{t_{1,2}}\left(0, r_{s}\right)=0$ and therefore $\omega_{l}\left(0, r_{s}\right)=\omega_{\mathrm{pl}}\left(r_{s}\right)$. But also in this case the virial theorem holds and assuming that $n\left(k, r_{s}\right)$ is a Gauß distribution, then Eq. (B1) turns out again. For phonons in Wigner crystals near melting see also Ref. 74.

\section{APPENDIX C: 1-MATRIX NEAR THE DIAGONAL AND FAR FROM IT}

The equation $\gamma\left(1 \mid 1^{\prime}\right)=\rho \delta_{\sigma_{1} \sigma_{1}^{\prime}} f\left(k_{\mathrm{F}}\left|\underline{r}_{1}-\underline{r}_{1}^{\prime}\right|\right)$ defines the dimensionless 1-matrix $f(x)$. Its small- $x$ behavior of Eq. (25) follows from the large- $k$ behavior of $n(k)$ [Eq. (5)]. Namely, with $\sin y / y=1-y^{2} / 3 !+y^{4} / 5 !-\cdots$ and with the integrability of $n(k) k^{2} k^{\nu}$ for $\nu=0, \ldots, 4$ it is

$$
f^{(\nu)}(0)=\left.\int_{0}^{\infty} d k^{3} n(k) k^{\nu}\left(\frac{d}{d y}\right)^{\nu} \frac{\sin y}{y}\right|_{y=0},
$$

which yields the first three terms of Eq. (25). Here $f^{(\nu)}(0)=\left.\left(\frac{d}{d x}\right)^{\nu} f(x)\right|_{x=0}$.

Because $n(k) k^{2} k^{5}$ is non-integrable, ope has to compute $f^{(5)}(0)$ with the Kimball procedure, 625 which defines by

$$
n(k)=\frac{C}{\left(1+k^{2}\right)^{4}}+\mathcal{N}(k), \quad C=\frac{8}{9 \pi^{2}}\left(\alpha r_{s}\right)^{2} g_{0}\left(r_{s}\right)
$$

a stronger (namely $\sim 1 / k^{10}$ for $k \rightarrow \infty$ ) decaying function $\mathcal{N}(k)$, so that $\mathcal{N}(k) k^{2} k^{5}$ is now integrable, yielding 0 because of $(d / d y)^{5} \sin y /\left.y\right|_{y=0}=0$. Thus

$$
f^{(5)}(0)=\left.\left(\frac{d}{d y}\right)^{5} p(x)\right|_{x=0}
$$

with

$$
p(x)=C \int_{0}^{\infty} \frac{d k^{3}}{\left(1+k^{2}\right)^{4}} \frac{\sin k x}{k x}=C \frac{\pi}{32}\left(3+3 x+x^{2}\right) \mathrm{e}^{-x} .
$$

It follows

$$
f^{(5)}(0)=-C \frac{\pi}{4}=-\frac{2}{9 \pi}\left(\alpha r_{s}\right)^{2} g_{0}\left(r_{s}\right),
$$

q.e.d.

The large- $x$ behavior (26) follows from Eq. (24) by partial integration. Thereby the discontinuities of $n\left(k, r_{s}\right)$ at $k \approx 1$ appear. They determine the amplitudes of the Friedel oscillations:

$$
f\left(x, r_{s}\right)=-z_{\mathrm{F}}\left(r_{s}\right) \frac{3 \cos x}{x^{2}}+z_{\mathrm{F}}\left(r_{s}\right) \frac{3 \sin x}{x^{3}}+f_{1}\left(x, r_{s}\right)
$$

with

$$
\begin{aligned}
f_{1}\left(x \gg 1, r_{s}\right)= & -A\left(r_{s}\right) \frac{3}{x^{3}}\left\{\left[\frac{\pi}{2}+\mathrm{Si}(x)\right] \cos x-\mathrm{Ci}(x) \times\right. \\
& \sin x\}+O\left(\frac{1}{x^{4}}\right) \\
= & -A\left(r_{s}\right) \pi \frac{3 \cos x}{x^{3}}+O\left(\frac{1}{x^{4}}\right) . \quad(\mathrm{C} 7)
\end{aligned}
$$

$z_{\mathrm{F}}\left(r_{s}\right)$ is the Fermi gap and $A\left(r_{s}\right)$ is the Fermi edge coefficient.

\section{APPENDIX D: FOCK COMPONENT OF THE STATIC STRUCTURE FACTOR AT SMALL $q$}

According to the definition of Eq. (32), the oscillations of $\left|f\left(x \gg 1, r_{s}\right)\right|^{2}$ [see Eq. (30)] only affect the discontinuities in $\tilde{n}^{2}\left(q, r_{s}\right)$ and in its derivatives at $q=2$, while 
the small- $q$ behavior of $\tilde{n}^{2}\left(q, r_{s}\right)$ is only affected by the oscillation-averaged part of $\left|f\left(x, r_{s}\right)\right|^{2}$, i.e., by

$\left\langle f^{2}\left(x \gg 1, r_{s}\right)\right\rangle=\frac{9}{2} \frac{z_{\mathrm{F}}^{2}\left(r_{s}\right)}{x^{4}}+9 \frac{\pi A\left(r_{s}\right) z_{\mathrm{F}}\left(r_{s}\right)}{x^{5}}+O\left(\frac{1}{x^{6}}\right)$.

Following the procedure of Kimball 6275 we define a function $\mathcal{F}(x)$ by

$$
\left\langle f^{2}\left(x, r_{s}\right)\right\rangle=\mathcal{F}(x)+\frac{9}{2} \frac{z_{\mathrm{F}}^{2}\left(r_{s}\right)}{\left(1+x^{2}\right)^{2}}+9 \frac{\pi A\left(r_{s}\right) z_{\mathrm{F}}\left(r_{s}\right)}{(1+x)^{5}}
$$

so that $\mathcal{F}(x \rightarrow \infty) \propto 1 / x^{6}$. Then, the second term will give the coefficient of the linear term in $\tilde{n}^{2}\left(q, r_{s}\right)$, while the third term will give the coefficient of a term $\propto q^{2} \ln q$. By carrying out the calculations one obtains Eq. (38), q.e.d. .
1 P. Fulde, Electron Correlation in Molecules and Solids (Springer, Berlin, 1995); N.H. March, Electron Correlation in Molecules and Condensed Phases (Plenum, New York, 1996).

2 R.J. Bartlett, Chemistry for the 21st Century (Wiley, Weinheim, 2001), p. 271.

3 E.R. Davidson, Reduced Density Matrices in Quantum Chemistry (Academic Press, New York, 1976).

${ }^{4}$ R. Erdahl and V.H. Jr. Smith, Density Matrices and Density Functionals (Reidel, Dortrecht, 1987).

5 A.J. Coleman and V.I. Yukalov, Reduced Density Matrices (Springer, Berlin, 2000).

6 J. Cioslowski, Many Electron Densities and ReducedDensity Matrices (Kluwer/Plenum, New York, 2000).

7 P. Ziesche, Solid State Commun. 82, 597 (1992).

8 P. Ziesche, in Ref. 6, p. 33.

9 J. P. Perdew, Electronic Structure of Solids '91, edited by P. Ziesche and H. Eschrig (Akademie Verlag, Berlin 1991); J. P. Perdew, K. Burke, and M. Ernzerhof, Phys. Rev. Lett. 77, 3865 (1996); 78, 1396 (1997).

10 S. H. Vosko, L. Wilk, and M. Nusair, Can. J. Phys. 58, 1200 (1980).

11 J. P. Perdew and A. Zunger, Phys. Rev. B 23, 5048, (1981).

12 J.P. Perdew and Y. Wang, Phys. Rev. B 45, 13244 (1992).

13 D. M. Ceperley and B. J. Alder, Phys. Rev. Lett. 45, 566 (1980).

14 J. P. Perdew and Y. Wang, Phys. Rev. B 46, 12947 (1992); 56, 7018 (1997).

15 P. Gori-Giorgi, F. Sacchetti, and G.B. Bachelet, Phys. Rev. B 61, 7353 (2000); 66, 159901(E) (2002).

16 P. Gori-Giorgi and J. P. Perdew, Phys. Rev. B, 66, 165118 (2002).

17 M. Corradini, R. Del Sole, G. Onida, and M. Palummo, Phys. Rev. B 57, 14569 (1998).

18 S. Moroni, D. M. Ceperley, and G. Senatore, Phys. Rev. Lett. 69, 1837 (1992); 75, 826 (1995).

19 C. F. Richardson and N. W. Ashcroft, Phys. Rev. B 50, 8170 (1994).

20 J. Cioslowski, P. Ziesche, and K. Pernal, Phys. Rev. B 63, 205105 (2001).

21 J. Cioslowski, P. Ziesche, and K. Pernal, J. Chem. Phys. 115, 8725 (2001).

22 J. Cioslowski, K. Pernal, and P. Ziesche, J. Chem. Phys. 117, 9560 (2002).

${ }^{23}$ K. Yasuda, Phys. Rev. Lett. 88, 053001 (2002).

${ }^{24}$ P. Ziesche, Int. J. Quantum Chem., 90, 342 (2002).

${ }^{25} \mathrm{P}$. Ziesche, in Electron Correlations and Materials Properties II edited by A. Gonis, N. Kioussis, and M. Ciftan
(Kluwer/Plenum, New York), in press.

26 A. W. Overhauser, Can. J. Phys. 73, 683 (1995).

27 P. Gori-Giorgi and J. P. Perdew, Phys. Rev. B 64, 155102 (2001).

28 B. Davoudi, M. Polini, R. Asgari, and M.P. Tosi, Phys. Rev. B 66, 075110 (2002). In a subsequent paper (condmat/0206456) the same authors go beyond the Hartree-like theory and show how the inclusion of exchange let emerge liquid-like structures with increasing coupling strength $r_{s}$ through the formation of a first-neighbor shell and further oscillations in the PD $g\left(x, r_{s}\right)$.

29 L. Lam and P.M. Platzman, Phys. Rev. B 9, 5122 (1974); G.E.W. Bauer, Phys. Rev. B 27, 5912 (1983); G.E.W. Bauer and J.R. Schneider, Phys. Rev. Lett. 52, 2061 (1984); A. Görling, M. Levy, and J.P. Perdew, Phys. Rev. B 47, 1167 (1993); S. Huotari et. al., Phys. Rev. B 66, 085104 (2002).

30 P. Ziesche, phys. stat. sol. (b), 232, 231 (2002).

31 I.O. Kulik, Z. Eksp. Teor. Fiz. 40, 1343 (1961) [Sov. Phys. JETP 13, 946].

32 N. H. March, Phys. Rev. 110, 604 (1958).

${ }^{33}$ Y. Takada and H. Yasuhara, Phys. Rev. B 44, 7879 (1991).

34 Y. Takada, private communication on the basis of the method described in Ref. 33.

35 G. Ortiz and P. Ballone, Phys. Rev. B 50, 1391 (1994); 56, 9970 (1997).

36 G. Senatore, S. Moroni, and D.M. Ceperley, in Physics of strongly coupled plasmas edited by W. D. Kraeft (World Scientific, Singapore, 1995).

37 B. Barbiellini and A. Bansil, J. Phys. Chem. Solids 62, 2181 (2001).

38 B. Farid, V. Heine, G.E. Hengel, and I.J. Robertson, Phys. Rev B 48, 11602 (1993).

39 The infinite slope at the Fermi edge was previously taken into meount in a study of the momentum distribution of ${ }^{3} \mathrm{He} ; \mathrm{t3}^{3}$ the authors also extracted the Fermi edge coefficient $A$ of Eqs. (6) and (7) from QMC simulations. At the equilibrium density they found $A=0.06(2)$.

40 J. Cioslowski, private communication.

41 H. Hellmann, Einführung in die Quantenchemie, Deuticke, Leipzig, 1937, pp. 61, 285 (the original russian version is of 23 October 1936: G. Gel'man, Quantenchemie, ONTI, Moscow and Leningrad, p. 428); R. P. Feynman, Phys. Rev. 56, 340 (1939). What usually is referred to as Hellmann-Feynman theorem has been first formulated by P. Güttinger, Z. Phys. 73, 169 (1932), see his Eq.(11). This result is already implicitly contained in Eq. (28) of M. Born and V. Fock, Z. Phys. 51, 165 (1928). Whereas in 
these papers any parameter is considered, Hellmann and later Feynman explicitly referred to the special case of nuclear coordinates within the Born-Oppenheimer approximation, leading to the 'Hellmann-Feynman' forces upon nuclei. Within perturbation theory the theorem was already given by E. Schrödinger, Ann. Phys. (Leipzig) 80, 437 (1926). Thus the theorem is due to Schrödinger, Born, Fock, Güttinger, Hellmann, and Feynman.

42 E. Daniel and S.H. Vosko, Phys. Rev. 120, 2041 (1960).

43 S. Moroni, G. Senatore, and S. Fantoni, Phys. Rev. B 55, 1040 (1997).

44 W. Macke, Z. Naturforsch. A5, 192 (1950).

45 M. Gell-Mann and K. A. Brueckner, Phys. Rev. 106, 364 (1957).

46 L. Onsager, L. Mittag, and M. J. Stephen, Ann. Phys. (Leipzig) 18, 71 (1966).

47 J. C. Kimball, J. Phys. A 8, 1513 (1975).

48 H. Yasuhara and Y. Kawazoe, Physica A 85, 416 (1976).

49 D.J.W. Geldart, Can. J. Phys. 453139 (1967).

50 J. C. Kimball, Phys. Rev. B 14, 2371 (1976).

51 Comparison of Eq. (A2) with Eq. (6) shows that RPA fails to reproduce the correct large- $k$ expansion of $n\left(k, r_{s}\right)$ : for small- $r_{s}$, instead of having $k^{8} n(k) \rightarrow \frac{8}{9} \pi^{2}\left(\alpha r_{s}^{2}\right)\left[\frac{1}{2}+O\left(r_{s}\right)\right]$, $\mathrm{RPA}$ yields $k^{8} n(k) \rightarrow \frac{8}{9} \pi^{2}\left(\alpha r_{s}^{2}\right)\left[1+O\left(r_{s}\right)\right]$. The lowest order exchange diagram 46 repairs this, see Figs. 1c and $1 \mathrm{f}$ of Ref. 24.

52 D. Pines and P. Nozières, Theory of Quantum Liquids (Benjamin, New York, 1966).

53 R. Sartor and C. Mahaux, Phys. Rev. C 21, 1546 (1980).

54 G. Ortiz, M. Harris, and P. Ballone, Phys. Rev. Lett. 82, 5317 (1999).

55 M. Seidl, J.P. Perdew, and S. Kurth, Phys. Rev. A 62, 012502 (2000); Phys. Rev. Lett. 84, 5070 (2000).

${ }^{56}$ F.H. Zong, C. Lin, and D.M. Ceperley, Phys. Rev. E 66, 036703 (2002).

57 P. Ziesche, Int. J. Quantum Chem. 56, 363 (1995).
58 P. Ziesche, J. Mol. Structure (Theochem) 527, 35 (2000) and references therein. Therein factors $1 / 2$ are erroneously incorporated in the definition of $g_{\uparrow \uparrow}(x)$ and $g_{\uparrow \downarrow}(x)$ such that $g_{\uparrow \uparrow}(\infty)=g_{\uparrow \downarrow}(\infty)=1 / 2$ instead of 1 .

$59 s(0)=0$ together with the Macke formula $\epsilon_{\mathrm{corr}}\left(r_{s}<<1\right)=$ $0.06218 \ln r_{s}+\ldots$ obviously contradicts Colling conjecture "correlation entropy $\sim$ correlation energy" .57,50

${ }^{60}$ D.M. Collins, Z. Naturforsch. A: Phys. Sci. 48a, 68 (1993).

61 M.B. Ruskai, J. Math. Phys. 11, 3218 (1970); K. Yasuda, Phys. Rev. A 63, 032517 (2001).

62 J. C. Kimball, Phys. Rev. A 7, 1648 (1973).

63 P.-O. Löwdin, Adv. Chem. Phys. 2, 207 (1959).

64 N. Iwamoto, Phys. Rev. A 33, 1940 (1986).

${ }^{65}$ P. Ziesche, in Electron Correlations and Materials Properties, edited by A. Gonis, N. Kioussis, and M. Ciftan (Kluwer/Plenum, New York, 1999).

66 P. Ziesche, J. Tao, M. Seidl, and J. P. Perdew, Int. J. Quantum Chem. 77, 819 (2000).

67 K. Fuchs, Proc. Roy. Soc. A 151, 585 (1935).

68 R.A., Coldwell-Horsfall and A.A. Maradudin, J. Math. Phys. 1, 395 (1960); 4, 582 (1963).

69 W. J. Jr. Carr, Phys. Rev. 122, 1437 (1961).

70 W. J. Jr. Carr, R.A. Coldwell-Horsfall, and A.E. Fein, Phys. Rev. 124, 747 (1961).

71 G. Diener, Diploma Thesis TH Dresden (1961), cf. Ref. 73, p. 19.

72 N.H. March and S. Sampanthar, Acta Phys. Hung. XIV, 61 (1962).

73 P. Ziesche and G. Paasch, in Ergebnisse in der Elektronentheorie der Metalle edited by P. Ziesche and G. Lehmann (Akademie/Springer, Berlin, 1983).

74 M.P. Tosi, in Electron Correlation in the Solid State, edited by N.H. March (Imperial College Press, London, 1999), p. 33

75 A. K. Rajagopal, J. C. Kimball, and M. Banerjee, Phys. Rev. B 18, 2339 (1978). 